\title{
Gua Lou Gui Zhi decoction attenuates post-stroke spasticity via the modulation of $\mathrm{GABA}_{\mathrm{B}}$ receptors
}

\author{
XIAOQIN ZHU ${ }^{1,2}$, HAIXIA HU ${ }^{1,2}$, ZUANFANG LI ${ }^{1,2}$, RUHUI LIN $^{1,2}$, JINGJIE MAO $^{1,2}$ and LIDIAN CHEN ${ }^{3,4}$ \\ ${ }^{1}$ Academy of Integrative Medicine Biomedical Research Center; ${ }^{2}$ Fujian Key Laboratory of Integrative Medicine on Geriatrics; \\ ${ }^{3}$ Fujian Key Laboratory of Exercise Rehabilitation; ${ }^{4}$ National Rehabilitation Research Center of Traditional Chinese Medicine, \\ Fujian University of Traditional Chinese Medicine, Fuzhou, Fujian 350122, P.R. China
}

Received March 30, 2015; Accepted June 11, 2015

DOI: $10.3892 / \mathrm{mmr} .2015 .4207$

\begin{abstract}
The aim of the present study was to investigate the mechanisms underlying the neuroprotective and antispastic effects of Gua Lou Gui Zhi decoction (GLGZD) in a rat model of middle cerebral artery occlusion (MCAO). The MCAO rats were treated with GLGZD (14.3 g/kg body weight) once a day for a period of seven days. Neurological deficit scores and screen tests were analyzed every other day. Following treatment with GLGZD for 7 days, the ischemic infarct volume of the rat brains was measured using 2,3,5-triphenyl tetrazolium chloride staining. Reverse transcription-polymerase chain reaction was performed in order to determine the mRNA expression levels of $\gamma$-amino butyric acid $B\left(\gamma-G_{A B A}\right)$ receptor $(R)$ in the cortical infarct region. Furthermore, the protein expression levels of $\mathrm{GABA}_{\mathrm{B}} \mathrm{R}$ were detected in the cortical infarct region by western blot analysis. Following 7 days, treatment with GLGZD significantly ameliorated the neurological defects and cerebral infarction in the MCAO rats. In addition, treatment with GLGZD ameliorated motor performance in the MCAO rats, as determined by screen tests. Furthermore, GLGZD was able to upregulate the mRNA and protein expression levels of $\mathrm{GABA}_{\mathrm{B} 1} \mathrm{R}$ and $\mathrm{GABA}_{\mathrm{B} 2} \mathrm{R}$ in the ischemic cerebral cortex. The results of the present study suggested that GLGZD may exert neuroprotective and antispastic effects in a cerebral ischemia model, through upregulating the expression of $\mathrm{GABA}_{B} \mathrm{R}$.
\end{abstract}

\section{Introduction}

Stroke is a common cause of disability and is the second leading cause of mortality in adults worldwide (1). One of the common

Correspondence to: Dr Lidian Chen, National Rehabilitation Research Center of Traditional Chinese Medicine, Fujian University of Traditional Chinese Medicine, 1 Qiuyang Road, Shangjie, Minhou, Fuzhou, Fujian 350122, P.R. China

E-mail: cld@fjtcm.edu.cn

Key words: Gua Lou Gui Zhi decoction, traditional Chinese medicine, cerebral ischemia, spasticity, $\gamma$-amino butyric acid B receptors symptoms of stroke is spasticity, which usually occurs within the first few days or weeks (2). The presence of spasticity one year after stroke has been reported in $\leq 38 \%$ of patients $(3,4)$. Spasticity is associated with pain, ankylosis, tendon retraction and muscle weakness in patients, all of which may limit rehabilitation $(5,6)$. Furthermore, spasticity interferes with the functional recovery of patients, and may eventually negatively affect the quality of life of stroke survivors (7). Therefore, it is essential to identify effective therapeutic approaches to treat spasticity, and to explore the mechanisms underlying spasticity, which are currently not well understood.

The possible pathogenesis of stroke-induced spasticity may be due to an excess of excitatory neurotransmitters, and a shortage of inhibitory neurotransmitters (8-10). This imbalance may be mediated by the excitatory neurotransmitter metabotropic glutamate receptor 1 (mGluR1) and the inhibitory neurotransmitter $\gamma$-amino butyric acid $\mathrm{B}\left(\gamma-\mathrm{GABA}_{\mathrm{B}}\right)$ receptor (R) (11). The $G_{A B A} R$ is composed of two subunits: $\mathrm{B} 1$ and $\mathrm{B} 2 \mathrm{GABA}_{\mathrm{B} 1}$ and $\left.\mathrm{GABA}_{\mathrm{B} 2}\right)(12)$, which have an important role in post-stroke spasticity (13). It has previously been shown that $\mathrm{GABA}_{\mathrm{B}} \mathrm{R}$ expression is reduced in the brain stems of rats with post-stroke spasticity (8), thus indicating that the $\mathrm{GABA}_{\mathrm{B}} \mathrm{R}$ is closely associated with stroke-induced spasticity. Furthermore, activation of $\mathrm{GABA}_{B} \mathrm{R}$ with baclofen, a GABA agonist, is able to effectively inhibit the muscle stretch reflex (14), suggesting that either restoring or preserving normal $\mathrm{GABA}_{\mathrm{B}} \mathrm{R}$ expression levels may be a potential approach to counteract stroke-induced spasticity. It has been hypothesized that various types of intervention, which are able to increase the expression of $\mathrm{GABA}_{\mathrm{B}} \mathrm{R}$, may relieve the symptoms of post-stroke spasticity.

Numerous pharmacological agents have been used to treat post-stroke spasticity, including baclofen, benzodiazepines and botulinum toxin (15-16). However, a number of these therapeutic drugs exhibit considerable side effects, including muscle weakness, hepatotoxicity, drowsiness and ataxia $(15,17,18)$. Therefore, there has recently been an increased focus on the use of natural products, such as traditional Chinese medicines (TCM), which have relatively low toxicity and fewer side effects, as compared with modern therapeutics. The classic TCM formula Gua Lou Gui Zhi decoction (GLGZD) was initially documented in 'Jin Gui Yao Lue', by Zhongjing Zhang during the Eastern 
Han Dynasty ( 25-220 AD). GLGZD consists of six herbs: Trichosanthes kirilowii Maxim, Paeonia lactiflora Pall, Cinnamomum cassia Presl, Glycyrrhiza uralensis Fisch, Zingiber officinale Rosc and Ziziphus jujuba Mill. As a well-known TCM compound, it has long been used to treat stroke-induced spasticity, epilepsy and spinal cord injury (19-21). The results of previous clinical experiments have demonstrated that GLGZD exerts a significant therapeutic effect on post-stroke disabilities, including muscular spasticity, by improving Fugl-Meyer and Barthel index scores (22). However, the precise mechanisms underlying the therapeutic effects of GLGZD remain largely unknown. In our previous study, GLGZD was shown to exert neuroprotective and antispastic effects in a cerebral ischemia model, via modulation of glutamate levels, and AMPA and NMDA glutamate receptors $(23,24)$. The $\mathrm{GABA}_{\mathrm{B}} \mathrm{R}$ controls neuronal excitability and glutamate receptor activity, which are associated with post-stroke spasms (25-27). The present study used a focal cerebral ischemia/reperfusion (I/R) injury rat model, which resembles human ischemic stroke, to evaluate the therapeutic efficacy of GLGZD against spasticity following cerebral ischemia. In addition, the underlying molecular mechanisms were investigated, which were hypothesized to be associated with the $\mathrm{GABA}_{\mathrm{B}} \mathrm{R}$.

\section{Materials and methods}

Reagents. Rabbit anti-rat polyclonal anti-GABA $\mathrm{B}_{1} \mathrm{R}$ (cat. no. 3835; dilution, 1:1,000), rabbit anti-rat polyclonal anti-GABA $_{\mathrm{B} 2} \mathrm{R}$ (cat. no. 3839; dilution, 1:1,000), rabbit anti-rat polyclonal anti- $\beta$-actin (cat. no. 4967; dilution, 1:1,000), and goat anti-rabbit horseradish peroxidase (HRP)-conjugated secondary antibodies (cat. no. 7074; dilution, 1:1,000) were obtained from Cell Signaling Technology, Inc. (Beverly, MA, USA). All other chemicals used in the present study, unless otherwise stated, were obtained from Sigma-Aldrich (St. Louis, MO, USA).

Preparation of water extract of GLGZD. GLGZD consists of six drugs: Trichosanthes kirilowii Maxim, Paeonia lactiflora Pall, Cinnamomum cassia Presl, Glycyrrhiza uralensis Fisch, Zingiber officinale Rosc and Ziziphus jujuba Mill, in a weight ratio of 10:3:3:2:3:3. Dried crude drugs were purchased from Guo Yi Tang Chinese Herbal Medicine store (Fujian, China), and the drug mixture was soaked in double distilled water for $30 \mathrm{~min}$. Subsequently, the mixture was decocted twice by boiling ( $1 \mathrm{~h}$ each time). The filtered solution from the two decoctions was then concentrated using a rotary evaporator (Model RE-2000; Yarong Biochemistry Instrument Factory, Shanghai, China), in order to obtain a final concentration of $1.06 \mathrm{~g} / \mathrm{ml}$. The GLGZD was subsequently stored for further analysis.

Animals. Male Sprague-Dawley rats $(n=27$; initial body weight, 240-280 g) were purchased from Shanghai Laboratory Animal Center (Shanghai, China), and housed in a temperature- and humidity-controlled room. The rats underwent a $12 \mathrm{~h}$ light/dark cycle and were provided access to food and water ad libitum. All animal treatments and experiments were approved by the Institutional Animal Care and Use Committee of Fujian University of Traditional Chinese Medicine (Fuzhou, China).

Focal cerebral I/R model and experimental groups. The cerebral I/R model was established following middle cerebral artery occlusion (MCAO), as described previously (28). Briefly, prior to surgery the rats were fasted for $24 \mathrm{~h}$; during this time the rats were still provided ad libitum access to water. Following anesthesia with $10 \%$ chloral hydrate by intraperitoneal injection $(300 \mathrm{mg} / \mathrm{kg}$ ), the left common carotid artery, left external carotid artery (ECA), and internal carotid artery (ICA) were exposed and isolated by a midline neck incision. Nylon surgical thread $(18-22 \mathrm{~mm})$ was inserted into the left ICA until the blunted distal end met resistance, in order to block the middle cerebral artery (MCA). A total of $2 \mathrm{~h}$ after occlusion, reperfusion was achieved by removing the thread to restore blood supply to the MCA. The rectal temperature of the rats was maintained at $37^{\circ} \mathrm{C}$ throughout the surgical procedure.

The rats were randomly divided into the following three groups: i) Sham-operated control (SC) group $(n=9)$, which underwent neck dissection and the exposure of ICA and ECA, without MCAO; ii) ischemia control (IC) group ( $n=9)$, which underwent MCAO surgery followed by reperfusion $2 \mathrm{~h}$ after occlusion; and iii) GLGZD group $(n=9)$, which underwent MCAO surgery followed by reperfusion $2 \mathrm{~h}$ after occlusion, and received GLGZD (14.3 $\mathrm{g} / \mathrm{kg}$ body weight) by intragastric administration once a day for a period of 7 days, as soon as the rats had recovered from reperfusion.

Scoring of neurological defects. A total of $2 \mathrm{~h}$ after induction of cerebral ischemia, and for the following 7 days, neurological defects of the rats were scored every other day. The neurological defects were scored by two researchers, who were blinded to the treatment conditions, according to a standard scoring system on a four-point scale (28): Score 0, no neurological defect; score 1, unable to fully extend the right forepaw; score 2, circling to the right; score 3, falling to the right; and score 4 , loss of walking ability. The rats that scored between 1 and 3 points were considered to be successful models.

Screen tests. In order to measure the muscle tone, strength, stamina and balance of the rats subjected to cerebral ischemia, screen tests were performed $2 \mathrm{~h}$ after induction of cerebral ischemia and for the following 7 days, using a net screen (Dashitong Equipment Co., Ltd., Fuzhou, China). The screen tests were conducted as described previously (29). The scoring criteria were as follows: 5, Holding onto the screen and climbing upward; 4, holding onto the screen with forelimbs and not falling down within $5 \mathrm{sec}$; 3 , holding onto the screen temporarily and slipping off a certain distance; 2 , falling to the ground within $5 \mathrm{sec}$; 1 , falling to the ground immediately, as soon as the screen was set at a vertical position.

Measurement of infarct volume. A total of 7 days after induction of cerebral ischemia, the rats were sacrificed with $10 \%$ chloral hydrate, and perfused transcardially with $0.9 \% \mathrm{NaCl}$. The brain of each rat was harvested and dissected into six coronal blocks $(2 \mathrm{~mm} /$ slice $)$. The fresh slices were incubated in $2 \%(\mathrm{w} / \mathrm{v})$ 2,3,5-triphenyltetrazolium chloride 
A

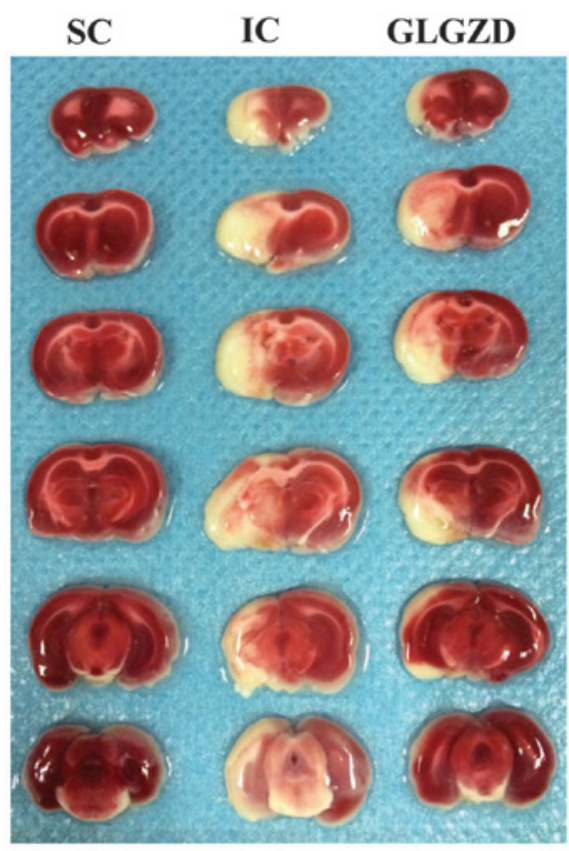

B

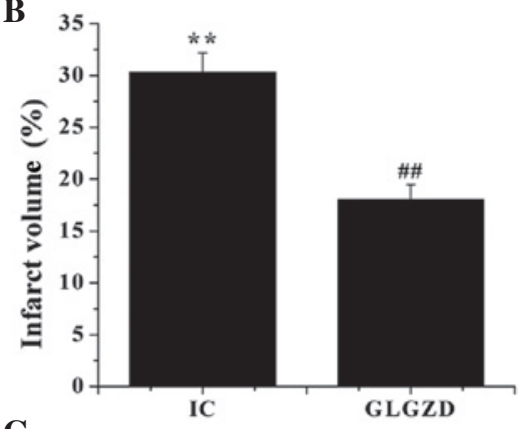

C

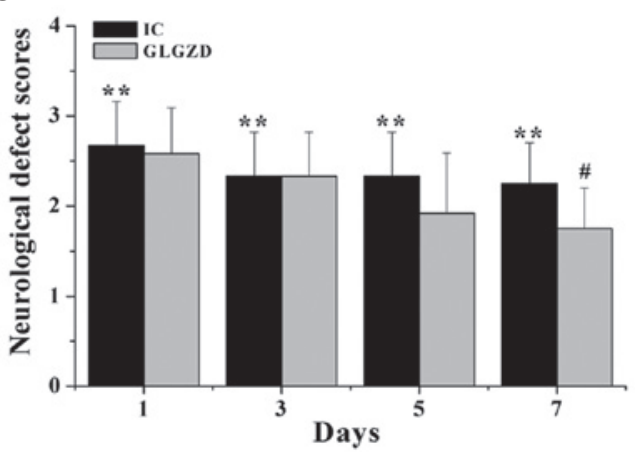

Figure 1. Effects of Gua Lou Gui Zhi decoction (GLGZD) on cerebral infarction and neurological defect scores in focal cerebral ischemia/reperfusion-injured rats. (A) At the end of the experiment, rat brain slices were stained with 2,3,5-triphenyltetrazolium chloride. Images are representative of three independent experiments. (B) Infarct volume was quantified as a percentage of the total brain volume, using the Motic Med 6.0 Digital Medical Image Analysis system. Data are presented as the mean \pm standard deviation from three individual rats in each group. ${ }^{* *} \mathrm{P}<0.01$, compared with the sham-operated control (SC) group; ${ }^{\# \# ~} \mathrm{P}<0.01$, compared with the ischemic control (IC) group. (C) Neurological defects were scored every other day in each group during the experiment. Data are presented as the mean \pm standard deviation from nine individual rats in each group. ${ }^{* *} \mathrm{P}<0.01$, compared with the $\mathrm{SC}$ group; ${ }^{*} \mathrm{P}<0.05$, compared with the IC group.

solution in phosphate-buffered saline for $20 \mathrm{~min}$ at $37^{\circ} \mathrm{C}$ in the dark. Normal areas of the brain were stained dark red based on intact mitochondrial function, whereas the infarct areas remained unstained. Images of the stained slices were captured using a high-resolution digital camera (Canon Sx20; Canon, Inc., Tokyo, Japan). The Motic Med 6.0 Digital Medical Image Analysis system (Motic Microscopes, Xiamen, China) was used to quantify the infarct volume by integration of the areas from the sections.

Reverse transcription-polymerase chain reaction (RT-PCR). Total RNA was extracted from the cortical infarct region of the rats using TRIzol ${ }^{\circledR}$ reagent (Invitrogen Life Technologies, Carlsbad, CA, USA), according to the manufacturer's instructions. Purified RNA ( $3 \mu \mathrm{g})$ was reverse transcribed into cDNA using the RevertAid ${ }^{\mathrm{TM}} \mathrm{H}$ Minus First Strand cDNA Synthesis kit (Fermentas, Thermo Fisher Scientific, Inc., Waltham, MA, USA), according to the manufacturer's instructions. The obtained cDNA was used to determine the mRNA expression levels of $\mathrm{GABA}_{\mathrm{B} 1} \mathrm{R}$ and $\mathrm{GABA}_{\mathrm{B} 2} \mathrm{R}$ by PCR using Taq DNA polymerase (Fermentas); $\beta$-actin served as an internal control. An S1000 PCR thermal cycler (Bio-rad Laboratories, Inc., Hercules, CA, USA) was used and the PCR conditions were as follows: $94^{\circ} \mathrm{C}$ for $3 \mathrm{~min}$, denaturation for $30 \mathrm{sec}$ at $94^{\circ} \mathrm{C}$ ( 35 cycles), annealing for $30 \mathrm{sec}$ at $58^{\circ} \mathrm{C}$, polymerization for $45 \mathrm{sec}$ at $72^{\circ} \mathrm{C}$ and finally extension for $10 \mathrm{~min}$ at $72^{\circ} \mathrm{C}$. The primers and the annealing temperatures $\left({ }^{\circ} \mathrm{C}\right)$ used for the amplification were as follows: Sense, 5'-CGG GTG GTA TGC TGA CAA CTG GT-3' (23 bp, 58 ${ }^{\circ} \mathrm{C}$ ) and antisense, $5^{\prime}$-ATG TTG GAA ATG CTT CGG GTG TT-3' (23 bp, 58 ${ }^{\circ} \mathrm{C}$ ) for
GABA $_{\mathrm{B} 1} \mathrm{R}$; sense, 5'-GGA CTT CAA CTA CAC AGA CCA CAC GC-3' (26 bp, 58 ${ }^{\circ} \mathrm{C}$ ) and antisense, 5'-TTG TAT TCG CCG ACC TTC ACC TCT CT-3' (26 bp, $\left.58^{\circ} \mathrm{C}\right)$ for $\mathrm{GABA}_{\mathrm{B} 2} \mathrm{R}$; and sense, 5'-CTA TCG GCA ATG AGC GGT TC-3' (20 bp, $58^{\circ} \mathrm{C}$ ) and antisense, 5'-ACT GTG TTG GCA TAG AGG TCT-3' (21 bp, $\left.58^{\circ} \mathrm{C}\right)$ for $\beta$-actin. Samples were analyzed by $1.5 \%$ agarose gel electrophoresis (Biowest, Hong Kong, China). The DNA bands were then examined using a Gel Documentation system (Gel Doc 2000; Bio-Rad Laboratories, Inc., Hercules, CA, USA).

Western blot analysis. The cortical infarct region of the rats was homogenized in non-denaturing lysis buffer (EMD Millipore, Boston, MA, USA) and centrifuged at $12,000 \mathrm{x}$ g for $10 \mathrm{~min}$. The protein concentration in the supernatants was then determined. Protein lysates were separated by $10 \%$ SDS-PAGE and then electrophoretically transferred to polyvinylidene fluoride membranes (Beyotime Institute of Biotechnology, Shanghai, China). The membranes were blocked for $2 \mathrm{~h}$ with $5 \%$ non-fat dry milk, and were then incubated with primary antibodies targeting $\mathrm{GABA}_{\mathrm{B} 1} \mathrm{R}, \mathrm{GABA}_{\mathrm{B} 2} \mathrm{R}$ and $\beta$-actin (1:1,000 dilution) overnight at $4^{\circ} \mathrm{C}$. The membranes were then incubated with appropriate HRP-conjugated secondary antibodies for $1 \mathrm{~h}$. The blots were visualized using enhanced chemiluminescence, and images were captured using a Bio-Image Analysis system (Bio-Rad Laboratories, Inc.).

Statistical analysis. All data are presented as the means of $\geq 3$ determinations. Statistical analysis of the data was performed with Student's t-test and one-way analysis of variance using the 


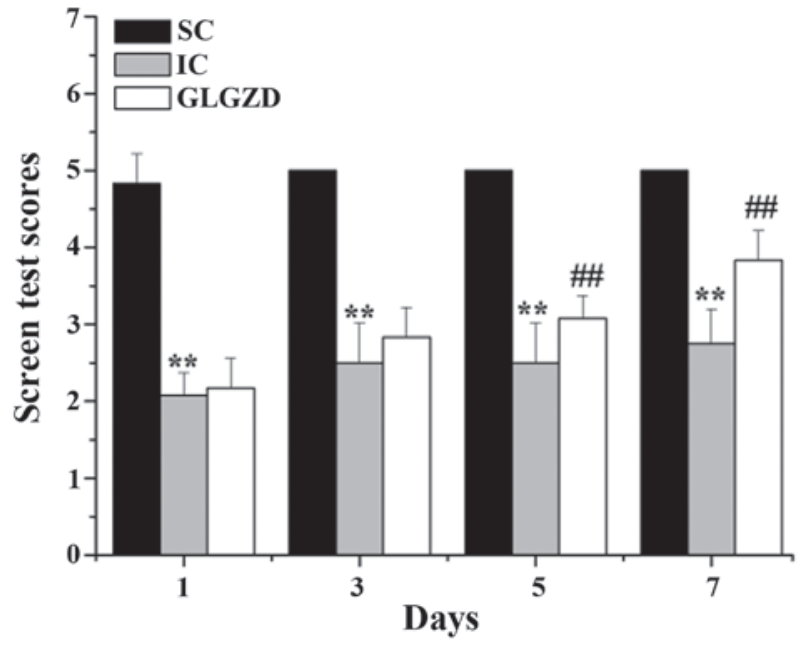

Figure 2. Effects of Gua Lou Gui Zhi decoction (GLGZD) on the screen test scores of focal cerebral ischemia/reperfusion injured rats $(n=9)$. Data are presented as the mean \pm standard deviation. ${ }^{* *} \mathrm{P}<0.01$, compared with the sham-operated control (SC) group; ${ }^{\# \#} \mathrm{P}<0.01$, compared with the ischemic control (IC) group.
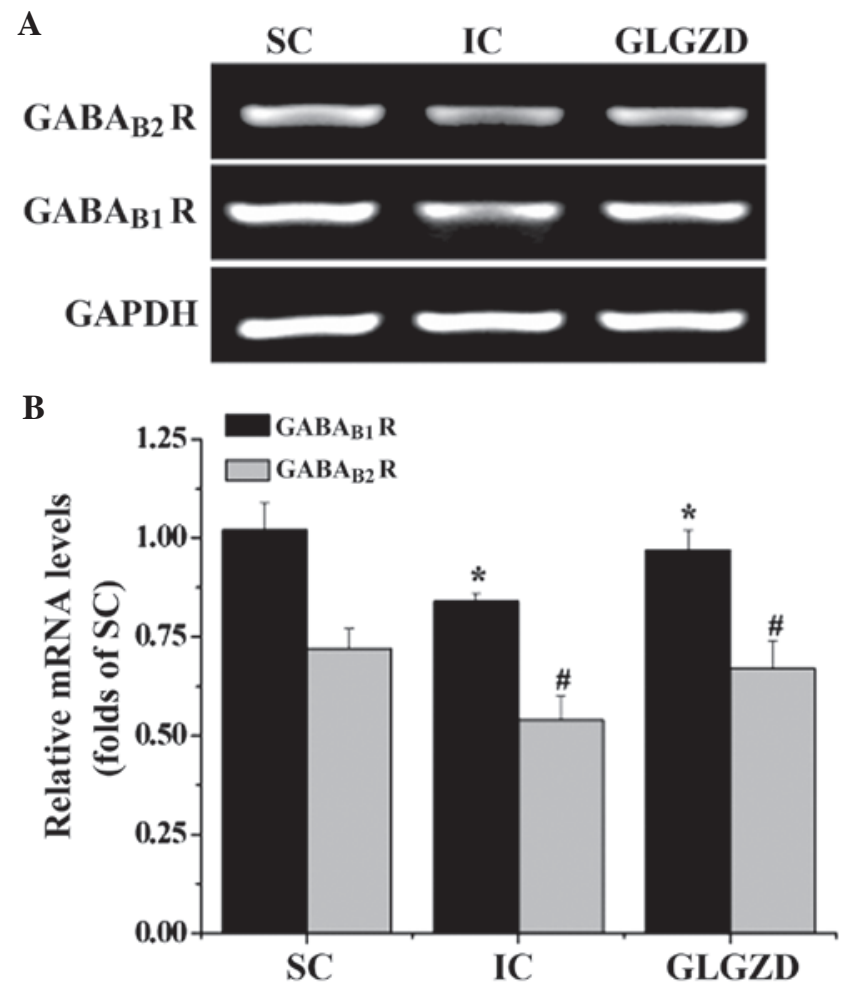

Figure 3. Effects of Gua Lou Gui Zhi decoction (GLGZD) on $\gamma$-amino butyric acid $\mathrm{B}\left(\mathrm{GABA}_{\mathrm{B}}\right)_{1}$ receptor $(\mathrm{R})$ and $\mathrm{GABA}_{\mathrm{B} 2} \mathrm{R}$ mRNA expression in the ischemic cerebral cortex of focal cerebral ischemia/reperfusion injured rats. (A) The mRNA expression levels of $G_{A B A} R$ and $G_{B A B} A_{B 2} R$ were determined by reverse transcription-polymerase chain reaction. $\beta$-actin was used as an internal control. Images are representative of three independent experiments. (B) Densitometric analysis. The data were normalized to the mean mRNA expression of the SC group. Data are presented as the mean \pm standard deviation. "P<0.05, compared with the sham-operated control (SC) group; ${ }^{\prime} \mathrm{P}<0.05$, compared with the ischemia control (IC) group.

SPSS package for Windows version 16.0 (SPSS Inc., Chicago, IL, USA). $\mathrm{P}<0.05$ was considered to indicate a statistically significant difference.
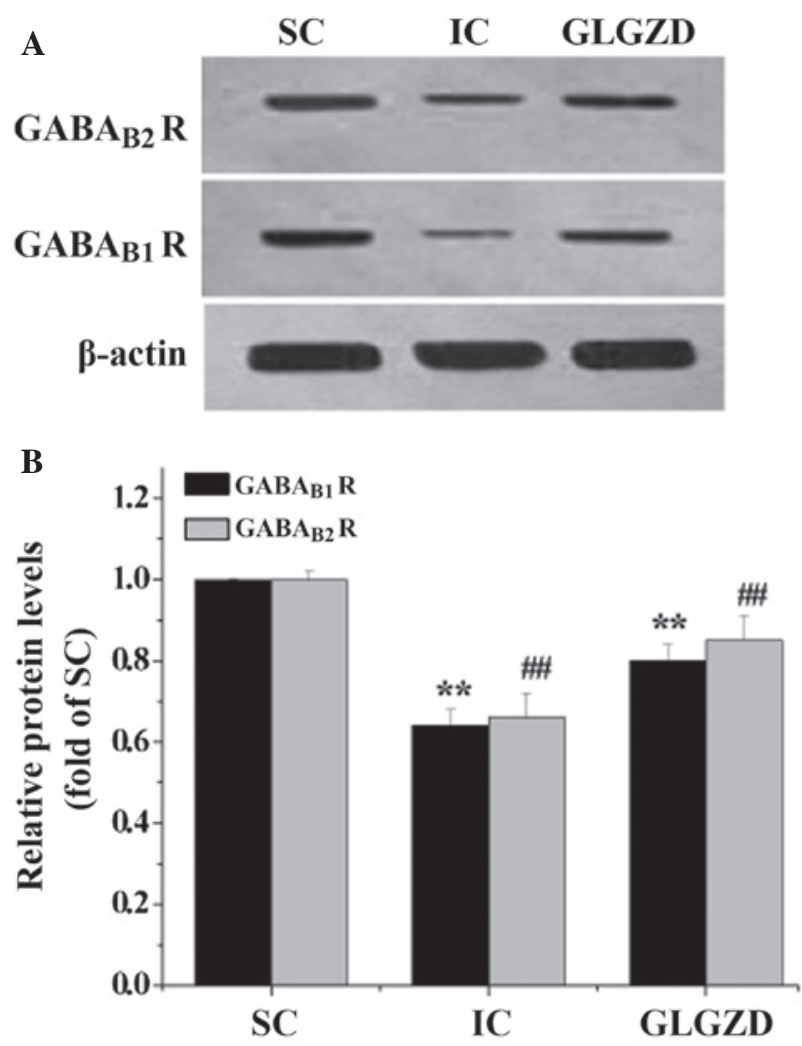

Figure 4. Effects of Gua Lou Gui Zhi decoction (GLGZD) on $\gamma$-amino butyric acid $\mathrm{B}\left(\mathrm{GABA}_{\mathrm{B}}\right)_{1}$ receptor $(\mathrm{R})$ and $\mathrm{GABA}_{\mathrm{B} 2} \mathrm{R}$ protein expression in the ischemic cerebral cortex of focal cerebral ischemia/reperfusion injured rats. (A) The protein expression levels of $\mathrm{GABA}_{\mathrm{B} 1} \mathrm{R}$ and $\mathrm{GABA}_{\mathrm{B} 2} \mathrm{R}$ were analyzed by western blotting. $\beta$-actin was used as an internal control. Images are representative of three independent experiments. (B) Densitometric analysis. The data were normalized to the mean protein expression of the $\mathrm{SC}$ group. Data are presented as the mean \pm standard deviation. ${ }^{* *} \mathrm{P}<0.01$, compared with the sham-operated control (SC) group. ${ }^{\# \#} \mathrm{P}<0.01$, compared with the ischemic control (IC) group.

\section{Results}

GLGZD ameliorates neurological defects and cerebral infarction. The neuroprotective effects of GLGZD were examined by evaluating the neurological defect scores and cerebral infarct volume of the rats. As shown in Fig. 1, all of the rats in the IC and GLGZD groups exhibited marked signs of cerebral infarction and neurological defects, whereas the rats in the SC group did not show any manifestation of cerebral injury. However, following treatment with GLGZD for 7 days, the cerebral infarct volumes were significantly reduced (Fig. 1A and B), and the neurological defect scores were improved (Fig. 1C). These results indicate that GLGZD may have therapeutic efficacy against cerebral I/R injury.

GLGZD improves motor performance. To determine the motor function of the rats subjected to cerebral I/R, screen tests were performed on the rats from all of the experimental groups. As shown in Fig. 2, the screen test scores of the IC rats were significantly decreased, as compared with the rats in the SC group. However, disordered motor function was significantly ameliorated following treatment with GLGZD between days 5 and 7. These results indicate that treatment with GLGZD may ameliorate cerebral I/R-induced spasticity in rats. 
$G L G Z D$ increases the expression levels of $G A B A_{B} R$ subunits following cerebral $I / R$. To further explore the mechanisms underlying the GLGZD-induced amelioration of post-cerebral I/R spasm, RT-PCR and western blot analysis were performed to detect the mRNA and protein expression levels of $\mathrm{GABA}_{B} \mathrm{R}$ subunits in the ischemic cortex, respectively. The mRNA and protein expression levels of the $\mathrm{GABA}_{\mathrm{B} 1} \mathrm{R}$ and $\mathrm{GABA}_{\mathrm{B} 2} \mathrm{R}$ were decreased in the IC group, as compared with the SC group. However, following treatment with GLGZD for 7 days, the expression levels of the $\mathrm{GABA}_{\mathrm{B} 1} \mathrm{R}$ and $\mathrm{GABA}_{\mathrm{B} 2} \mathrm{R}$ were increased (Fig. 3 and 4). These data suggest that treatment with GLGZD may promote the activation of the $\mathrm{GABA}_{\mathrm{B}} \mathrm{R}$ in the ischemic cortex.

\section{Discussion}

Post-stroke spasticity is one of the most physically debilitating conditions in aged populations, due to muscle hyperactivity, which results in limb stiffness and muscle spasm (30). It has been hypothesized that post-stroke spasticity is induced by an imbalance between excitatory and inhibitory neurotransmitters, which may be mediated by the expression of mGlu R1 and $\mathrm{GABA}_{\mathrm{B}} \mathrm{R}$. This imbalance may induce the abnormal transmission of sensory information and motion instruction, and subsequently increase the muscle activity and the active tonic stretch reflex (31). Direct evidence regarding the involvement of the $\mathrm{GABA}_{\mathrm{B}} \mathrm{R}$ in post-stroke spasticity has yet to be reported; however, numerous lines of research have suggested that the $\mathrm{GABA}_{\mathrm{B}} \mathrm{R}$ may affect the clinical signs of spasticity, and participate in an experimental animal model of cerebral ischemia-induced limb spasms $(8,32)$. A previous clinical study demonstrated that baclofen, a GABA agonist, was able to cross the blood-brain barrier and bind to the $\mathrm{GABA}_{\mathrm{B}} \mathrm{R}$ at the presynaptic terminal, suggesting that it may be used for the treatment of spasticity (14). In addition, catgut implantation at acupoints has been shown to relieve limb spasticity in the brain stem of rats after stroke via upregulation of the $\mathrm{GABA}_{\mathrm{B}} \mathrm{R}$ expression (8). These studies suggest that the $\mathrm{GABA}_{\mathrm{B}} \mathrm{R}$ may have an important role in the mechanisms underlying post-stroke spasticity. The $\mathrm{GABA}_{\mathrm{B}} \mathrm{R}$ is a heterodimeric G-protein-coupled receptor, which is composed of two subunits: $\mathrm{GABA}_{\mathrm{B} 1} \mathrm{R}$ and $\mathrm{GABA}_{\mathrm{B} 2} \mathrm{R}$. The $\mathrm{GABA}_{\mathrm{B}} \mathrm{R}$ is abundantly expressed post- and presynaptically throughout the central nervous system in virtually all types of neurons (33). It mediates slow inhibitory neurotransmission, and/or hyperpolarizes the neuronal membrane, thereby controlling the excitability of neurons and consequently participating in post-stroke spasticity (34-36).

GLGZD is a classical TCM formula that has previously been used to treat post-stroke spasticity. Recent clinical trials have demonstrated that GLGZD had a significant therapeutic effect on limb spasm in patients following cerebral stroke $(21,22)$. However, the mechanisms underlying its neuroprotective and antispastic effects remain largely unknown. The present study aimed to further elucidate the mechanisms of GLGZD, using a focal cerebral ischemia rat model. The results of the present study demonstrated that treatment with GLGZD exerted neuroprotective effects by improving neurological defects and reducing cerebral infarct volume. In addition, GLGZD was shown to improve motor performance, as determined using a screen test. Furthermore, as expected,
GLGZD was able to significantly upregulate the mRNA and protein expression levels of both $\mathrm{GABA}_{\mathrm{B} 1} \mathrm{R}$ and $\mathrm{GABA}_{\mathrm{B} 2} \mathrm{R}$ in the cerebral ischemic cortex.

In conclusion, the present study demonstrated that treatment with GLGZD exerts neuroprotective effects and improves spasticity in an ischemic stroke model via upregulation of the $\mathrm{GABA}_{\mathrm{B}} \mathrm{R}$. These findings suggest that GLGZD may be a potential therapeutic agent for the treatment of cerebral ischemia and spasticity.

\section{Acknowledgements}

The present study was supported by the Ministry of Science and Technology of the International Science and Technology Cooperation Projects (grant no. 2011DFG33240), and the Nature Science Foundation of Fujian Province of China (grant no. 2014J01358).

\section{References}

1. Donnan GA, Fisher M, Macleod M and Davis SM: Stroke. Lancet 371: 1612-1623, 2008.

2. Mayer NH and Esquenazi A: Muscle overactivity and movement dysfunction in the upper motoneuron syndrome. Phys Med Rehabil Clin N Am 14: 855-883, 2003.

3. Sommerfeld DK, Eek EU, Svensson AK, Holmqvist LW and von Arbin MH: Spasticity after stroke: Its occurrence and association with motor impairments and activity limitations. Stroke 35: 134-139, 2004

4. Lundström E, Terént A and Borg J: Prevalence of disabling spasticity 1 year after first-ever stroke. Eur J Neurol 15: 533-539, 2008.

5. Duncan PW, Zorowitz R, Bates B, Choi JY, Glasberg JJ, Graham GD, Katz RC, Lamberty K and Reker D: Management of Adult Stroke Rehabilitation Care: A clinical practice guideline. Stroke 36: e100-e143, 2005.

6. Brown P: Pathophysiology of spasticity. J Neurol Neurosurg Psychiatry 57: 773-777, 1994.

7. Doan QV, Brashear A, Gillard PJ, Varon SF, Vandenburgh AM, Turkel CC and Elovic EP: Relationship between disability and health-related quality of life and caregiver burden in patients with upper limb poststroke spasticity. PM R 4: 4-10, 2012.

8. Liu CM, Li RQ, Song XL and Feng XD: Effect of catgut implantation at acupoints on GABAB and mGluR1 expressions in brain stem of rats with spasticity after stroke. J Tradit Chin Med 34: 566-571, 2014.

9. Ellenberger C, Mevissen M, Doherr M, Scholtysik G and Jaggy A: Inhibitory and excitatory neurotransmitters in the cerebrospinal fluid of epileptic dogs. Am J Vet Res 65: 1108-1113, 2004.

10. Ramanathan M, Babu CS, Justin A and Shanthakumari S: Elucidation of neuroprotective role of endogenous GABA and energy metabolites middle cerebral artery occluded model in rats. Indian J Exp Biol 50: 391-397, 2012.

11. Yang JP, Zhang XQ, Wang XD and Wang KW: Inhibitory and excitatory amino acids in cerebral spinal fluid in children with cerebral palsy. Zhong Hua Xiao Er Wai Ke Za Zhi 19: 282-284, 1998 (In Chinese).

12. Galvez T, Parmentier ML, Joly C, Malitschek B, Kaupmann K, Kuhn R, Bittiger H, Froestl W, Bettler B and Pin JP: Mutagenesis and modeling of the GABAB receptor extracellular domain support a venus flytrap mechanism for ligand binding. J Biol Chem 274: 13362-13369, 1999.

13. Benke D: GABAB receptor trafficking and interacting proteins: targets for the development of highly specific therapeutic strategies to treat neurological disorders? Biochem Pharmacol 86: 1525-1530, 2013.

14. Goldstein EM: Spasticity management: An overview. J Child Neurol 16: 16-23, 2001.

15. Lapeyre E, Kuks JB and Meijler WJ: Spasticity: Revisiting the role and the individual value of several pharmacological treatments. NeuroRehabilitation 27: 193-200, 2010.

16. Simon O and Yelnik AP: Managing spasticity with drugs. Eur J Phys Rehabil Med 46: 401-410, 2010. 
17. Sun X: Research on formula treating paralysis and spasticity from 'treatise on febrile and miscellaneous diseases'. Zhongguo Zhong Yi Ji Chu Yi Xue Za Zhi 8: 644-645, 2010 (In Chinese).

18. Yelnik AP, Simon O, Bensmail D, Chaleat-Valayer E, Decq P, Dehail P, Quentin V, Marque P, Parratte B, Pellas F, et al; Agence française de sécurité sanitaire des produits de santé: Drug treatments for spasticity. Ann Phys Rehabil Med 52: 746-756, 2009.

19. Satkunam LE: Rehabilitation medicine: 3. Management of adult spasticity. CMAJ 169:1173-119, 2003.

20. Zhang L and Ai H: Effects of Gua Lou Gui Zhi decotion on c-fos and c-jun in epileptic rats. Chuan Hua Xi Zhong Yi Yao Yan Jiu Suo 23: 21-22, 2005. (In Chinese).

21. Yang CM, Chen LD and Tao J: New usage of a classical formula-Gua Lou Gui Zhi decotion. Liao ning Zhong Yi Za Zhi 8: 166-167, 2012 (In Chinese).

22. Chen YL, Chen LD, Tao J: Clinical research on treating limbs spam from cerebral apoplexy with Gualou Guizhi decoction. Clin J Chin Med 5: 7-9, 2013 (In Chinese).

23. Huang J, Tao J, Xue XH, Yang S, Han P, Lin Z, Xu W, Lin J, Peng $\mathrm{J}$ and Chen L: Gua Lou Gui Zhi decoction exerts neuroprotective effects on post-stroke spasticity via the modulation of glutamate levels and AMPA receptor expression. Int J Mol Med 31: 841-848, 2013.

24. Chen X, Li H, Huang M, Huang M, Xu W, Chu K, Chen L and Zhang Y: Effect of Gua Lou Gui Zhi decoction on focal cerebral ischemia-reperfusion injury through regulating the expression of excitatory amino acids and their receptors. Mol Med Rep 10: 248-254, 2014

25. Chalifoux JR and Carter AG: GABAB receptor modulation of synaptic function. Curr Opin Neurobiol 21: 339-344, 2011.
26. Oshiro M, Hefferan MP, Kakinohana O, Lukacova N, Sugahara K, Yaksh TL and Marsala M: Suppression of stretch refex activity after spinal or systemic treatment with AMPA receptor antagonist NGX424 in rats with developed baclofen tolerance. Br J Pharmacol 161: 976-985, 2010.

27. Gómez-Soriano J, Goiriena E and Taylor J: Spasticity therapy reacts to astrocyte GluA1 receptor upregulation following spinal cord injury. Br J Pharmacol 161: 972-975, 2010.

28. Longa EZ, Weinstein PR, Carlson S and Cummins R: Reversible middle cerebral artery occlusion without craniectomy in rats. Stroke 20: 84-91, 1989.

29. Guo J, Liu L, Ma C, Xu B, Duan X and Wang B: Effect of restraint stress on depression-like behaviors in rats after transient focal cerebral ischemic injury. Neural Regen Res 2: 390-394, 2007.

30. Mayer NH, Esquenazi A and Childers MK: Common patterns of clinical motor dysfunction. Muscle Nerve (Suppl 6): S21-S35, 1997.

31. Doble A: The role of excitotoxicity in neurodenenerative disease: Implications for therapy. Pharmacol Ther 81: 163-221, 1999.

32. Bowery NG: GABAB receptor: a site of therapeutic benefit .Curr Opin Pharmacol 6: 37-43, 2006.

33. Bettler B, Kaupmann K, Mosbacher J and Gassmann M: Molecular structure and physiological functions of GABA(B) receptors. Physiol Rev 84: 835-867, 2004.

34. Gassmann $M$ and Bettler B: Regulation of neuronal GABA(B) receptor functions by subunit composition. Nat Rev Neurosci 13: 380-394, 2012.

35. Chalifoux JR and Carter AG: GABAB receptor modulation of synaptic function. Curr Opin Neurobiol 21: 339-344, 2011.

36. Benarroch EE: GABAB receptors: Structure, functions, and clinical implications. Neurology 78: 578-584, 2012. 